\title{
Efficacy of generic oral directly acting agents in patients with hepatitis $\mathrm{C}$ virus infection
}

\author{
S. Gupta ${ }^{1}$ \\ G. Rout ${ }^{1}$ \\ A. H. Patel ${ }^{2}$ \\ M. Mahanta ${ }^{1}$ \\ N. Kalra ${ }^{1}$ \\ P. Sahu ${ }^{1}$ \\ R. Sethia ${ }^{1}$ \\ A. Agarwal $^{1}$ \\ G. Ranjan ${ }^{1}$ \\ S. Kedia ${ }^{1}$ \\ S. K. Acharya ${ }^{1}$ \\ B. Nayak ${ }^{1}$ \\ Shalimar ${ }^{1}$ (1)
}

${ }^{1}$ Department of Gastroenterology, All India Institute of Medical Sciences, New Delhi, India

${ }^{2}$ Division of Gastroenterology and Hepatology, Department of Internal

Medicine, University of Michigan Medical

School, Ann Arbor, MI, USA

Correspondence

Shalimar, Department of Gastroenterology, All India Institute of Medical Sciences, New Delhi, India.

Email: drshalimar@gmail.com

\section{Summary}

Novel direct-acting antivirals (DAAs) are now the standard of care for the management of hepatitis $\mathrm{C}$ virus (HCV) infection. Branded DAAs are associated with high sustained virological response at 12 weeks post-completion of therapy (SVR12), but are costly. We aimed to assess the efficacy of generic oral DAAs in a real-life clinical scenario. Consecutive patients with known $\mathrm{HCV}$ infection who were treated with generic-oral DAA regimens (May 2015 to January 2017) were included. Demographic details, prior therapy and SVR12 were documented. Four hundred and ninety patients (mean age: $38.9 \pm 12.7$ years) were treated with generic DAAs in the study time period. Their clinical presentations included chronic hepatitis (CHC) in 339 (69.2\%) of cases, compensated cirrhosis in 120 (24.48\%) cases and decompensated cirrhosis in 31 (6.32\%) cases. Genotype 3 was most common ( $n=372,75.9 \%)$ followed by genotype $1(n=97,19.8 \%)$. Treatment naïve and treatment-experienced (defined as having previous treatment with peginterferon and ribavirin) were $432(88.2 \%)$ and 58 (11.8\%), respectively. Generic DAA treatment regimens included sofosbuvir in combination with ribavirin $(n=175)$, daclatasvir alone $(n=149)$, ribavirin and peginterferon $(n=80)$, ledipasvir alone ( $n=43)$, daclatasvir and ribavirin $(n=37)$, and ledipasvir and ribavirin $(n=6)$. Overall SVR12 was $95.9 \%$ (470/490) for all treatment regimens. SVR12 for treatment naïve and experienced patients was $97.0 \%$ (419/432) and $87.9 \%$ (51/58), respectively, $P=.005$. High SVR12 was observed with various regimens, irrespective of genotype and underlying liver disease status. There were no differences in SVR12 with 12 or 24 weeks therapy. No major adverse event occurred requiring treatment stoppage. Generic oral DAAs are associated with high SVR rates in patients with HCV infection in a real-life clinical scenario.

\section{KEYWORDS}

chronic hepatitis C, cirrhosis, RAV, SVR12, treatment

Abbreviations: CAP, controlled attenuation parameter; CHC, chronic hepatitis C; DAA, directly acting antivirals; HCV, hepatitis C virus; LSM, liver stiffness measurement; MELD, model for endstage liver disease; OR, odds ratio; SVR12, sustained virological response at 12 weeks; WHO, world health organization. 


\section{1 | INTRODUCTION}

Hepatitis $\mathrm{C}$ virus (HCV) infection poses a significant public health concern with an estimated $2 \%-3 \%$ overall global prevalence, and 0.9\%-1.9\% prevalence in India. ${ }^{1}$ The vast majority of patients (up to $80 \%$ ) who have HCV develop chronic hepatitis that can progress to chronic liver disease, cirrhosis and hepatocellular carcinoma. Novel direct-acting antivirals (DAAs) are now the standard of care for the management of HCV infection. Moreover, recent HCV management guidelines recommend that all patients positive for $\mathrm{HCV}$ RNA be considered for therapy irrespective of the serum alanine aminotransferase levels and underlying liver disease status. ${ }^{2,3}$ The World Health Organization (WHO) recently launched an initiative to eliminate viral hepatitis by 2030 ; and in order to achieve these goals, approximately 71 million HCV-positive patients need to be treated, mostly from low-income countries of Asia and Africa. ${ }^{4}$

In interferon-free clinical trials, branded DAAs are associated with high sustained virological response at 12 weeks after completion of treatment (SVR12). ${ }^{5-7}$ The cost of these branded drugs differs globally across countries and is out of reach in most developing countries, where treatment expenses are borne by the patients. Currently, in the United States, branded ledipasvir/sofosbuvir combination therapy (brand name Harvoni) costs approximately $\$ 1000$ USD a pill, amounting to greater than $\$ 80000$ USD for a 12 -week course of treatment. Gilead Sciences Inc, USA had given voluntary manufacturing licenses to several Indian companies including Cadila Zydus Ltd., Cipla Ltd., and Natco Pharma Ltd for generic production of sofosbuvir or its combinations with ledipasvir in 2014. ${ }^{8}$ Indian generic manufacturers including Cipla Ltd., and Natco Pharma Ltd., also obtained sublicenses in 2015 for generic production of daclatasvir through Bristol-Meyers Squibb by way of the Medicines Patent Pool. ${ }^{9}$ There are limited data on the efficacy of these generic brands across genotypes and varied clinical conditions. The aim of this study was to assess the efficacy of generic oral DAAs in a real-life clinical scenario and to compare efficacy across different treatment regimens, hepatitis $C$ genotypes and severity of liver disease.

\section{PATIENTS AND METHODS}

\subsection{Inclusion and exclusion criteria}

In this prospective study, all consecutive HCV-positive patients evaluated in the Department of Gastroenterology at the All India Institute of Medical Sciences (AIIMS), in New Delhi, India between May 2015 and January 2017 were included. Written informed consent was obtained from all patients. The study was approved by the institute's ethics committee. Patients with co-infection with hepatitis $B$ virus (HBV) and human immunodeficiency virus (HIV), hepatocellular carcinoma (HCC), pregnancy and lactation, active tuberculosis, malignancy, Wilson's disease, chronic kidney disease and those not willing to consent were excluded from the study. All demographic, virological and SVR12 data were collected from a prospectively maintained database.

\section{2 | Patient evaluation}

All patients underwent a complete blood count (CBC), liver function tests (LFT), kidney function tests, fasting blood sugar and an abdominal ultrasound. Upper gastrointestinal endoscopy and triple phase CT of the abdomen were performed in patients with concern for cirrhosis and hepatocellular carcinoma. In patients with suspected autoimmune liver disease and Wilson's disease workup was performed as per the standard guidelines. ${ }^{10,11}$ Serological testing of all patients for HBV surface antigen, anti-HCV antibody and HIV-1 and 2 was carried out using commercial ELISA. For HCV genotyping and quantitation, viral nucleic acid was extracted using an automated nucleic acid isolation system (Qiasymphony, Qiagen). The HCV genotyping was carried out using the AmpliSensò HCV-genotype-FRT PCR kit which can detect genotypes 1-6.

High viral load was defined as $\geq 600000 \mathrm{IU} / \mathrm{mL}$, and low viral load was defined as $<600000 \mathrm{IU} / \mathrm{mL}$. HCV RNA quantification was performed at baseline, end of therapy and 12 weeks posttreatment (SVR12). SVR12 was achieved if HCV RNA was negative/below detectable limit. While all patients had HCV RNA levels checked at baseline, end of treatment and SVR12, several patients had HCV viral loads checked at additional intervals. The diagnosis of cirrhosis was based on histologic findings on liver biopsy, fibroscan showing a liver stiffness $\geq 12.5 \mathrm{KPa}$ (Echosens, France) or a combination of conventional endoscopic (varices, gastric antral vascular ectasia or portal hypertensive gastropathy on esophagogastroduodenoscopy) and imaging criteria (CT scan or ultrasound findings concerning for cirrhosis). Decompensated cirrhosis was defined as the presence of jaundice, ascites, variceal haemorrhage or hepatic encephalopathy. The model for end-stage liver disease (MELD) score $^{12}$ was calculated at baseline and after 12 weeks of completion of treatment. Patients were classified as being either "treatment naïve" with no prior treatment given or "treatment experienced" if they had attempted a prior regimen including peginterferon and ribavirin. Breakthrough was defined as undetectable HCV RNA during treatment followed by the appearance of HCV RNA, despite continued treatment. All patients who were included in the study had a fibroscan performed prior to initiation of DAAs, as per our institutional protocol.

\section{3 | Management protocol}

The management protocol of chronic hepatitis $\mathrm{C}(\mathrm{CHC})$ patients was as per the American Association for the Study of Liver Diseases (AASLD) practice guidelines for management of HCV infection. ${ }^{3}$ Sofosbuvir remained the backbone of all combination therapies. Initially, when sofosbuvir alone was available, a combination of sofosbuvir with ribavirin alone or in combination with peginterferon was used for both HCV genotypes 1 and 3. Later, as other DAAs became commercially available in India, sofosbuvir was used in combination with daclatasvir (for genotypes 2, 3, 5 and those with genotype not available) and ledipasvir (for genotype 1 and 4). The use of ribavirin was considered in patients who previously relapsed or did not respond to interferon-based regimens, 
and in patients with cirrhosis; the duration of therapy in these patients was 24 weeks as combination therapy with sofosbuvir. Patients were followed until the end of therapy and then had follow-up studies for a further 12 weeks. Various DAA combination drugs were purchased by patients with prescriptions. Many patients $(n=220)$ were not able to afford the medications and received the generic drugs (sofosbuvir and ribavirin, with or without peginterferon) free of cost from Cadila Zydus Ltd. and Cipla Ltd., at the request of the treating physicians. HCV genotyping and viral load estimation was performed free of cost for all patients.

\section{4 | Statistical analysis}

The normally distributed variables were expressed as mean \pm standard deviation (SD) and continuous variables with skewed distribution as median (inter-quartile range). Categorical data are presented as frequency and percentage. Univariate analysis was performed to assess the factors associated with SVR12 using an independent $t$ test or Mann-Whitney $U$ test for continuous variables. The chi-square test or Fisher's exact test for categorical variables was used whenever applicable. The continuous variables were dichotomized to assess the effect on SVR. A P value of .05 was considered as statistically significant. Data were analysed using IBM SPSS Statistics software (version 20.0, Chicago, IL, USA).

\section{3 | RESULTS}

\section{1 | Baseline characteristics}

A total of $683 \mathrm{HCV}$-positive patients were evaluated during the study period and recommended to initiate DAA therapy. Of these patients, 193 were excluded because of an inability to afford therapy or incomplete follow-up after the 1st visit $(n=65)$, ongoing therapy $(n=90)$, chronic kidney disease $(n=28)$, co-infection with HBV $(n=8)$ and HIV ( $n=2)$ (Figure 1). Of the 490 patients included (mean age $38.9 \pm 12.7$ years), clinical presentations included chronic hepatitis ( $n=339,69.2 \%$ ), compensated cirrhosis $(n=120,24.5 \%$ ) and decompensated cirrhosis ( $n=31,6.3 \%$ ). Genotype 3 was most common in 372 (75.9\%) patients followed by genotype 1 in 97 (19.8\%). There were 432 (88.2\%) treatment naïve and 58 (11.8\%) treatment-experienced (defined as previous treatment with peginterferon and ribavirin) patients. Pretreatment high viral load was observed in 242 (49.4\%) patients. The clinical and demographic details are shown in Table 1.

\section{2 | SVR according to genotype, underlying liver disease and type of regimen}

Overall SVR12 was seen in 95.9\% (470/490). SVR12 for treatmentnaïve and treatment-experienced patients was 97.0\% (419/432) and $87.9 \%$ (51/58), respectively, $P=.005$.

\begin{tabular}{|c|c|c|c|c|c|c|}
\hline & & \multicolumn{3}{|c|}{ Total no. of HCV-positive patients treated, $n=683$} & \multirow{2}{*}{\multicolumn{2}{|c|}{$\begin{array}{l}\text { Reasons for exclusion }(n=193) \\
\text { ot affording therapy/ lost to follow- } \\
\text { up after } 1^{\text {st }} \text { visit }(n=65) \\
\text { Presently on therapy }(n=90) \\
\text { Hepatitis B coinfection }(n=8) \\
\text { HIV coinfection }(n=2) \\
\text { Chronic kidney disease }(n=28)\end{array}$}} \\
\hline & & \multicolumn{3}{|c|}{ Total no. of HCV-positive patients included, $\mathrm{n}=490$} & & \\
\hline & \multicolumn{5}{|c|}{$\begin{array}{l}\text { Genotype } 1(n=97), 2(n=3), 3(n=372), 4(n=11), 5(n=2) \text { and NOS }(n=5) \\
\text { CHC }(n=339) \text {, cirrhosis }(n=120) \text {, and decompensated cirrhosis }(n=31)\end{array}$} & \\
\hline & \multicolumn{3}{|c|}{ Genotype 1, SVR12 } & \multicolumn{3}{|c|}{ Genotype 3, SVR12 } \\
\hline Regimens & CHC & Cirrhosis & $\begin{array}{l}\text { Decompensated } \\
\text { cirrhosis }\end{array}$ & CHC & Cirrhosis & $\begin{array}{l}\text { Decompensated } \\
\text { cirrhosis }\end{array}$ \\
\hline $\mathrm{SOF}+\mathrm{RBV}$ & $18 / 19(94.7 \%)$ & $7 / 8(100 \%)$ & $10 / 10(100 \%)$ & $77 / 78(98.7 \%)$ & $43 / 43(100 \%)$ & $11 / 12(91.7 \%)$ \\
\hline $\begin{array}{l}\mathrm{SOF}+\mathrm{RBV}+ \\
\text { Peginterferon }\end{array}$ & $13 / 13(100 \%)$ & $1 / 1(100 \%)$ & - & $48 / 51(94.1 \%)$ & $11 / 11(100 \%)$ & - \\
\hline $\mathrm{SOF}+\mathrm{DAC}$ & $1 / 1(100 \%)$ & - & - & $117 / 120(97.5 \%)$ & $15 / 16(93.8 \%)$ & $4 / 4(100 \%)$ \\
\hline $\mathrm{SOF}+\mathrm{DAC}+\mathrm{RBV}$ & - & - & - & $6 / 8(75.0 \%)$ & $23 / 24(95.8 \%)$ & $5 / 5(100 \%)$ \\
\hline $\mathrm{SOF}+\mathrm{LDV}$ & $28 / 31(90.3 \%)$ & $7 / 8(87.5 \%)$ & - & - & - & - \\
\hline $\mathrm{SOF}+\mathrm{LDV}+\mathrm{RBV}$ & $1 / 2(50 \%)$ & $3 / 4(75.0 \%)$ & - & - & - & - \\
\hline SVR12 & $61 / 66(92.4 \%)$ & $18 / 21(85.7 \%)$ & $10 / 10(100 \%)$ & $248 / 257(96.5 \%)$ & $92 / 94(97.9 \%)$ & $20 / 21(95.2 \%)$ \\
\hline
\end{tabular}

FIGURE 1 SVR12 in various combinations of oral directly acting agents. HCV, hepatitis C virus; CHC, chronic hepatitis C; HIV, human immunodeficiency virus; NOS, not otherwise specified; SVR12, sustained viral response at 12 wks; SOF, Sofosbuvir; RBV, ribavirin; DAC, daclatasvir; LDV, ledipasvir 
TABLE 1 Baseline demographic and clinical characteristics of patients

\begin{tabular}{|c|c|}
\hline Variable & Overall $(n=490)$ \\
\hline Age (y) & $38(28-48)$ \\
\hline Sex (male:female), n (\%) & 281 (57.3\%): 209 (42.7\%) \\
\hline BMI $\left(\mathrm{kg} / \mathrm{m}^{2}\right)$ & $22.7(20.2-26.0)$ \\
\hline Fasting blood glucose (mg/dL) & $92(85-103)$ \\
\hline Total cholesterol (mg/dL) & $150(127-182)$ \\
\hline Vitamin D3 (ng/mL) & $20.9(12.8-31.4)$ \\
\hline TSH (mIU/L) & $1.9(1.3-3.0)$ \\
\hline Alpha-fetoprotein (ng/mL) & $3.9(2.3-6.9)$ \\
\hline $\begin{array}{l}\text { Baseline liver stiffness } \\
\text { measurement (LSM, KPa) }\end{array}$ & $7.3(5.3-15.4)$ \\
\hline $\begin{array}{l}\text { Baseline LSM interquartile range } \\
\text { (IQR) }\end{array}$ & $1.2(0.6-2.2)$ \\
\hline $\begin{array}{l}\text { Baseline controlled attenuation } \\
\text { parameter (CAP) }\end{array}$ & $213(177-255)$ \\
\hline $\begin{array}{l}\text { Baseline CAP interquartile range } \\
\text { (IQR) }\end{array}$ & $38.0(26-55)$ \\
\hline Baseline HCV RNA (IU/mL) & 528000 (100000-3142800) \\
\hline HCV RNA (<600 $000 \mathrm{IU} / \mathrm{mL})$ & $248 / 490(50.6 \%)$ \\
\hline HCV RNA ( $\geq 600000 \mathrm{IU} / \mathrm{mL}$ ) & $242 / 490(49.4 \%)$ \\
\hline Haemoglobin (g/dL) & $13.1(11.8-14.7)$ \\
\hline Total Leucocytes Count (per $\mathrm{mm}^{3}$ ) & $6800(5300-8200)$ \\
\hline Platelets Count $\left(\times 10^{3} / \mathrm{mm}^{3}\right)$ & $170(120-220)$ \\
\hline Bilirubin (mg/dL) & $0.6(0.5-1.0)$ \\
\hline Aspartate aminotransferase (IU/L) & $57(38-93)$ \\
\hline Alanine aminotransferase (IU/L) & $63(40-110)$ \\
\hline Alkaline phosphatase (IU/L) & $203(153-267)$ \\
\hline Total protein (g/dL) & $7.4(7.1-7.8)$ \\
\hline Serum albumin $(\mathrm{g} / \mathrm{dL})$ & $4.5(4.0-4.9)$ \\
\hline International normalized ratio & $1.02(1.0-1.1)$ \\
\hline Blood urea (mg/dL) & $23(19-30)$ \\
\hline Serum creatinine $\mathrm{mg} / \mathrm{dL}$ & $0.8(0.7-0.9)$ \\
\hline \multicolumn{2}{|l|}{ Type of Liver disease } \\
\hline Chronic hepatitis C & $339 / 490$ (69.2\%) \\
\hline Compensated cirrhosis & $120 / 490(24.5 \%)$ \\
\hline Decompensated cirrhosis & $31 / 490(6.3 \%)$ \\
\hline \multicolumn{2}{|l|}{ Prior therapy received } \\
\hline Naïve & $432 / 490$ (88.2\%) \\
\hline $\begin{array}{l}\text { Prior relapse and non- } \\
\text { responder (Peg-Interferon and } \\
\text { Ribavirin) }\end{array}$ & $58 / 490$ (11.8\%) \\
\hline \multicolumn{2}{|l|}{ Hepatitis C Genotype } \\
\hline Genotype 1 & 97 (19.8\%) \\
\hline Genotype 2 & $3(0.6 \%)$ \\
\hline Genotype 3 & $372(75.9 \%)$ \\
\hline Genotype 4 & $11(2.2 \%)$ \\
\hline Genotype 5 & $2(0.4 \%)$ \\
\hline Not otherwise specified & $5(1.0 \%)$ \\
\hline
\end{tabular}

All values are expressed as $n$ (interquartile range) or $n(\%)$, unless otherwise specified.
The details of various combinations using sofosbuvir are shown in Figure 1. The SVR12 in genotype $1 \mathrm{CHC}$ and cirrhosis were $92.4 \%(61 / 66)$ and $85.7 \%(18 / 21)$, respectively. Only six genotype 1 patients received the sofosbuvir plus ledipasvir plus ribavirin regimen. Among these, the SVR12 in CHC and cirrhosis were 50\% (1/2) and 75\% (3/4), respectively.

With the various sofosbuvir-based regimens, SVR12 was $95.8-100 \%$ in genotype 3 cirrhosis and $91.7-100 \%$ in decompensated cirrhosis. The SVR12 in genotype $3 \mathrm{CHC}$ was $97.5 \%(177 / 120)$ in the sofosbuvir plus daclatasvir regimen and $75 \%(6 / 8)$ in the sofosbuvir plus daclatasvir plus ribavirin regimen. SVR12 rates observed with various regimens, according to genotype and underlying liver disease status are shown in Figure 1 and Table 2. There were no differences in the SVR12 with 12 weeks and 24 weeks therapy (Table 3). The details of SVR12 in different sofosbuvir-based regimens, according to genotype 1 and 3 , underlying liver disease and duration of therapy, are shown in Tables S1 and S2.

\section{3 | Predictors of SVR12}

In univariate analysis, treatment-experienced patients (relapsers and nonresponders to peginterferon and ribavirin) had a lower SVR12 (87.9\%) as compared to treatment-naïve patients (97.0\%), odds ratio (OR) $0.226,95 \% \mathrm{Cl}(0.086-0.593)$. The rates of SVR12 with the treatment regimen of sofosbuvir, ledipasvir and ribavirin in combination therapy was $66.7 \%(4 / 6)$ and lower as compared to other regimens (>90\%). No differences were found among patients who achieved or those who did not achieve SVR12 (Table 4), in terms of factors related to the virus (including genotype, viral load) or host factors (including age, sex, presence of diabetes, body mass index, underlying liver disease status ( $\mathrm{CHC}$, cirrhosis and decompensated cirrhosis), liver stiffness measurement (LSM) or controlled attenuation parameter (CAP) on fibroscan\} or duration of therapy (12 vs 24 weeks). Multivariate analysis for predictors of SVR12 was not carried out due to small number of patients in the treatment regimen consisting of sofosbuvir, ledipasvir and ribavirin. There was no change in SVR12 when the analysis was performed after excluding patients who were lost to follow-up.

\subsection{Patients lost to follow-up and relapsers}

Seven patients (including one breakthrough) were documented to have relapsed on the basis of positive HCV RNA after 12 weeks of therapy. Thirteen treatment-naïve patients were lost to follow-up and were treated as nonresponders when analysing outcomes (total $n=20$ ). There were no treatment-experienced patients who were lost to follow-up. All relapsers to sofosbuvir-based therapy were treatment-experienced and had previously received peginterferon and ribavirin-based therapy. The characteristics of patients lost to follow-up and relapsers according to underlying liver disease are shown in Table S3. The details of patients lost to follow-up and relapsers according to underlying liver disease and genotype are shown in Tables S4 and S5, respectively. There were no differences in genotype, viral load, age, diabetes, BMI, fibroscan values, CAP, underlying liver disease status, duration of therapy and treatment regimen used. 
TABLE 2 SVR12 According to the Genotype and underlying liver disease

\begin{tabular}{lrrrr} 
& CHC (SVR12) & Cirrhosis (SVR12) & $\begin{array}{l}\text { Decompensated } \\
\text { cirrhosis (SVR12) }\end{array}$ & P value \\
\hline Genotype 1 & $61 / 66(92.4 \%)$ & $18 / 21(85.7 \%)$ & $10 / 10(100 \%)$ & .377 \\
Genotype 2 & $2 / 2(100 \%)$ & $1 / 1(100 \%)$ & & - \\
\hline Genotype 3 & $248 / 257(96.5 \%)$ & $92 / 94(97.9 \%)$ & $20 / 21(95.2 \%)$ & .747 \\
Genotype 4 & $8 / 8(100 \%)$ & $3 / 3(100 \%)$ & - & - \\
\hline Genotype 5 & $2 / 2(100 \%)$ & - & - & - \\
\hline NOS & $4 / 4(100 \%)$ & $1 / 1(100 \%)$ & - & - \\
\hline Total $(\mathrm{n}=490)$ & $325 / 339(95.9 \%)$ & $115 / 120(95.8 \%)$ & $30 / 31(96.8 \%)$ & .969 \\
\hline
\end{tabular}

NOS, not otherwise specified
TABLE 3 SVR12 rates according to the duration of therapy

\begin{tabular}{cllll} 
& \multicolumn{2}{l}{ SVR12 } & & \\
\cline { 2 - 4 } & CHC & Cirrhosis & $\begin{array}{l}\text { Decompensated } \\
\text { Cirrhosis }\end{array}$ & P value \\
\cline { 2 - 3 } $\begin{array}{c}12 \text { wks therapy } \\
(n=264)\end{array}$ & $217 / 227(95.4 \%)$ & $32 / 34(94.1 \%)$ & $2 / 3(66.7 \%)$ & 0.068 \\
\hline $\begin{array}{c}24 \text { wks therapy } \\
(n=226)\end{array}$ & $108 / 112(95.9 \%)$ & $83 / 86(96.5 \%)$ & $28 / 28(100 \%)$ & 0.600 \\
\hline Total $(n=490)$ & 339 & 120 & 31 & \\
\hline
\end{tabular}

\section{5 | Change in MELD score at 12 weeks post- therapy}

The baseline model for end-stage liver disease (MELD) score in patients with compensated cirrhosis at baseline was $8.5 \pm 2.4$ and at end of 12 weeks of treatment was $8.4 \pm 2.4 ; P=.672$. MELD score in patients with decompensated cirrhosis at baseline was $10.3 \pm 3.5$ and at end of 12 weeks of treatment was $10.3 \pm 3.4 ; P=.957$.

\section{6 | Adverse events}

No major adverse events requiring treatment stoppage occurred. Among the 357 patients with available paired samples (at baseline and after therapy), $37 / 357$ (10.4\%) had worsening anaemia with a haemoglobin below $10 \mathrm{~g} / \mathrm{dL}$.

\section{DISCUSSION}

In this study, we observed an overall SVR12 of 95.9\%; with higher rates in treatment-naïve patients as compared to those who were treatment-experienced. This study includes a large number of patients who received generic DAA treatment for HCV genotype 3 infection, which is highly prevalent in India. A paradigm shift from interferon-based therapies to interferon-free regimens has occurred in the management of HCV due to the introduction of oral DAAs. Recent guidelines advocate treatment with oral DAAs for all HCV patients who have no contraindications. ${ }^{2,3}$ Moreover, the World Health Organization recently launched a drive to eliminate viral hepatitis by $2030 .{ }^{13}$ Hepatitis C eradication can be best achieved globally by treatment with DAAs; however, the major barrier continues to remain the cost of treatment. The cost of treatment largely depends on the region of treatment and the availability of brand-name patented drugs; branded drugs are generally expensive. ${ }^{14}$ Several generic low-cost drugs of these DAAs are now available in developing countries. The introduction of low-cost generic brands-including sofosbuvir, ledipasvir and daclatasvir-has led to a reduction in the overall cost of therapy to as little as $\$ 300$ USD for a 12-week course of therapy.

Irrespective of underlying cirrhosis, SVR12 was very high in treatmentnaïve patients, while rates of SVR12 in treatment-experienced patients were lower. We previously reported an SVR ( 24 weeks) rate of $64 \%$ in patients treated with peginterferon and ribavirin in combination. ${ }^{15}$ In this present study, overall SVR12 was $95.9 \%$ (470/490), which is similar to those reported previously from other studies performed in the Indian subcontinent. ${ }^{16-18}$ Prior multicenter studies evaluating the efficacy of branded DAA have reported similar high SVR12 rates. ${ }^{5-7,19}$ Our study supports the fact that generic DAAs are associated with high efficacy.

A recent review on efficacy and safety of oral DAA reported an overall SVR12 of $92 \%$ in cirrhosis patients treated with sofosbuvir/ ledipasvir combination therapy. ${ }^{20}$ Other real-life observational studies have reported an SVR12 between $94 \%$ and $98 \%$ in treatment-naïve genotype 1 patients treated with combination of sofosbuvir and ledipasvir $^{21,22}$; including a study by Zeng et al, wherein naïve genotype 1 patients were treated with a combination of generic sofosbuvir and ledipasvir, SVR12 among cirrhotic and noncirrhotic patients was $96.8 \%$ and $96.9 \%$, respectively. The overall SVR12 in our study in genotype $1(90.3 \%)$ was lower than that reported from other studies. This may have been due, in part, to small sample size in this subgroup and classification of patients who were lost to follow-up as treatment failures. 
TAB LE 4 Univariate analysis of predictors of SVR12

\begin{tabular}{|c|c|c|}
\hline Factors & SVR12 & $P$ value \\
\hline \multicolumn{3}{|l|}{ Viral factors } \\
\hline \multicolumn{3}{|l|}{ Genotype } \\
\hline 1 & $89 / 97$ (91.8\%) & .317 \\
\hline 2 & $3 / 3(100 \%)$ & \\
\hline 3 & $360 / 372(96.8 \%)$ & \\
\hline 4 & $11 / 11(100 \%)$ & \\
\hline 5 & $2 / 2(100 \%)$ & \\
\hline NOS & $5 / 5(100 \%)$ & \\
\hline \multicolumn{3}{|l|}{ Viral load (IU/mL) } \\
\hline$\leq 600000$ & $240 / 248(96.8 \%)$ & .368 \\
\hline$>600000$ & $230 / 242(95.0 \%)$ & \\
\hline \multicolumn{3}{|l|}{ Host Factors } \\
\hline \multicolumn{3}{|l|}{ Age (y) } \\
\hline$\geq 40$ & $218 / 228(95.6 \%)$ & .821 \\
\hline$<40$ & $252 / 262(96.2 \%)$ & \\
\hline \multicolumn{3}{|l|}{ Sex } \\
\hline Male & $268 / 281$ (95.4\%) & .645 \\
\hline Female & $202 / 209$ (96.7\%) & \\
\hline \multicolumn{3}{|l|}{ Diabetes } \\
\hline Absent & $398 / 417$ (95.4\%) & 0.335 \\
\hline Present & $72 / 73(98.6 \%)$ & \\
\hline \multicolumn{3}{|l|}{ BMI $(n=335), \mathrm{kg} / \mathrm{m}^{2}$} \\
\hline$<23$ & $166 / 172(96.5 \%)$ & 1.000 \\
\hline$>23$ & $158 / 163(96.9 \%)$ & \\
\hline \multicolumn{3}{|l|}{ Liver disease } \\
\hline $\mathrm{CHC}$ & $325 / 339$ (95.9\%) & .969 \\
\hline Cirrhosis & $115 / 120(95.8 \%)$ & \\
\hline Decompensated Cirrhosis & $30 / 31$ (96.8\%) & \\
\hline \multicolumn{3}{|l|}{ LSM (n = 449), KPa } \\
\hline LSM $<6$ & $153 / 159(96.2 \%)$ & .578 \\
\hline $\mathrm{LSM} \geq 6$ & $282 / 290(97.2 \%)$ & \\
\hline \multicolumn{3}{|l|}{$\operatorname{CAP}(n=411)$} \\
\hline$C A P \geq 206 \mathrm{~dB} / \mathrm{m}$ & $232 / 238(97.5 \%)$ & .405 \\
\hline CAP $<206 \mathrm{~dB} / \mathrm{m}$ & $166 / 173(96.0 \%)$ & \\
\hline \multicolumn{3}{|c|}{ MELD (cirrhosis and decompensated cirrhosis) } \\
\hline MELD $<10$ & $114 / 118(96.6 \%)$ & .612 \\
\hline MELD $\geq 10$ & $31 / 33(93.9 \%)$ & \\
\hline \multicolumn{3}{|l|}{ Prior Therapy Received } \\
\hline Naïve & $419 / 432(97.0 \%)$ & .005 \\
\hline Peg-Interferon and Ribavirin & $51 / 58(87.9 \%)$ & \\
\hline \multicolumn{3}{|l|}{ Treatment type } \\
\hline \multicolumn{3}{|l|}{ Duration (wks) } \\
\hline 12 & $251 / 264(95.1 \%)$ & .365 \\
\hline 24 & $219 / 226(96.9 \%)$ & \\
\hline
\end{tabular}

Treatment regimen
TABLE 4 (Continued)

\begin{tabular}{|c|c|c|}
\hline Factors & SVR12 & $P$ value \\
\hline Sofosbuvir + Ribavirin & $171 / 175(97.7 \%)$ & .001 \\
\hline $\begin{array}{l}\text { Sofosbuvir + Ribavirin + Pegyl } \\
\text { ated Interferon }\end{array}$ & $77 / 80$ (96.3\%) & \\
\hline Sofosbuvir + Daclatasvir & $145 / 149(97.3 \%)$ & \\
\hline $\begin{array}{l}\text { Sofosbuvir + Daclatasvir + Rib } \\
\text { avirin }\end{array}$ & $34 / 37$ (91.9\%) & \\
\hline Sofosbuvir + Ledipasvir & $39 / 43$ (90.7\%) & \\
\hline $\begin{array}{l}\text { Sofosbuvir + Ledipasvir + Riba } \\
\text { virin }\end{array}$ & $4 / 6(66.7 \%)$ & \\
\hline
\end{tabular}

NOS, not otherwise specified; BMI, body mass index; $\mathrm{CHC}$, chronic hepatitis C; LSM, liver stiffness measurement; CAP, controlled attenuation parameter; MELD, model for end-stage liver disease.

After excluding such patients, our SVR12 rates were similar to those reported in other studies.

A study from Spain reported an overall SVR12 of 93.8\% in genotype 3 patients treated with sofosbuvir and daclatasvir combination therapy. ${ }^{23}$ Another study in Asian American patients with $\mathrm{CHC}$ and advanced liver disease (genotypes 1, 2, 3 and 6), treated with multiple oral DAA combinations, reported a similar overall SVR. ${ }^{24}$ The SVR12 in $\mathrm{CHC}$ patients treated with sofosbuvir plus daclatasvir and ribavirin was lower than that of other combinations, possibly due to a small sample size of eight patients in this subgroup. The SVR12 in compensated cirrhosis and decompensated cirrhosis in our study was similar to that reported in previous studies. ${ }^{25}$

We used various oral DAA drug combinations, as per the AASLD $\mathrm{HCV}$ management guidelines. ${ }^{3}$ Our data reinforce that the drug regimens recommended by AASLD are associated with high SVR. Prior treatment exposure was the only factor associated with virological failure, corroborating a previous study that involved genotype 4 patients and showed that male gender and prior treatment-experience (with peginterferon) were predictors of nonresponse. ${ }^{26}$

Our patients did not develop any major complications requiring stoppage of therapy. Anaemia developed in $10 \%$ of the patients and was secondary to ribavirin therapy. Prior studies have reported minor side effects with oral DAAs, most of which are not significant and do not mandate cessation of therapy. ${ }^{10}$ These observations provide reassurance that generic DAAs are associated with a similar safety profile as the branded DAAs. To provide cost-conscious care, HCV patients can be prescribed a complete course of therapy during the initial patient encounter without the need for repeated blood tests, as was the case with an interferon-based regimen.

We did not find any significant difference in the MELD score after 12 weeks of therapy as compared with baseline. This may be because the mean MELD score at baseline was low, and therefore, the fractional change was not significant. Prospective studies need to evaluate the effect of oral DAAs on the change in MELD and Child-Pugh-Turcotte scores.

Seven patients had relapse on sofosbuvir-based therapies. The aetiology of this is unclear as these patients had different treatment regimens. We did not assess for resistance associated variants in these patients. 
Similar to the paradigm shift in the management of human immunodeficiency virus (HIV) infection, where the use of generic drugs has led to significant reduction of cost of therapy, the use of generic medications for HCV infection will make drugs more affordable, especially in developing countries, where treatment costs are borne by the patients themselves. The availability of generics with good efficacy and tolerability has the potential to redefine the management and outcomes of HCV infection, and in the future, potentially eradicate it.

This study has a few limitations. The data were from a single tertiary care centre, which is associated with a referral bias. Another limitation is the observational design of the study, which was not randomized for drug regimens or HCV genotypes. Therefore, there is a selection bias for patient enrollment such as inclusion of larger numbers of HCV genotype 3 patients and differences in patient enrollment for the various generic drug combinations. Generic drugs have certain limitations, as compared to branded drugs. Branded drugs undergo extensive testing for quality, safety and efficacy. There is no post-marketing surveillance for generics. We used multiple generic brands; however, we did not compare head-to-head outcomes with different manufacturers. We included both treatment-naïve and treatment-experienced patients, as well as a spectrum of liver disease including chronic hepatitis $\mathrm{C}$, compensated and decompensated cirrhosis. We assessed virologic relapse by repeat documentation of the same genotype by real time PCR and not by phylogenetic analysis of nucleotide sequence, which would have accurately differentiated relapse from reinfection. In conclusion, generic oral directly acting agents are associated with high SVR rates in patients with HCV infection in a real-life clinical scenario.

\section{ACKNOWLEDGEMENTS}

Mr Amar Negi and Mr Anurag for maintenance of database. Cadila Zydus Ltd., and Cipla Ltd., for providing free sofosbuvir and ribavirin with or without peginterferon for 220 non-affording patients.

\section{CONFLICT OF INTERESTS}

None of the authors has any financial, professional or personal conflicts that are relevant to the manuscript.

\section{AUTHORSHIP STATEMENT}

Swatantra Gupta, Gyanranjan Rout, Mousumi Mahanta, Nancy Kalra, Pabitra Sahu, Rahul Sethia, Ashish Agarwal, Gyan Ranjan: data acquisition. Arpan H. Patel: draft writing. Saurabh Kedia, data acquisition, draft writing. Subrat Kumar Acharya, data acquisition, design. Baibaswata Nayak, draft writing, study concept. Shalimar: draft writing, study concept, design, statistical analysis

\section{ORCID}

A. H. Patel iD http://orcid.org/0000-0003-1239-9613

Shalimar (iD http://orcid.org/0000-0003-1247-437X

\section{REFERENCES}

1. Puri P. Tackling the hepatitis B disease burden in India. J Clin Exp Hepatol. 2014;4:312-319.

2. European Association for the Study of the Liver. EASL recommendations on treatment of hepatitis C 2016. J Hepatol. 2017;66:153-194.

3. AASLD/IDSA HCV Guidance Panel. Hepatitis C guidance: AASLDIDSA recommendations for testing, managing, and treating adults infected with hepatitis C virus. Hepatology. 2015;62:932-954.

4. Polaris Observatory HCV Collaborators. Global prevalence and genotype distribution of hepatitis $C$ virus infection in 2015: a modelling study. Lancet Gastroenterol Hepatol. 2017;2(3):161-176.

5. Foster GR, Pianko S, Brown A, et al. Efficacy of sofosbuvir plus ribavirin with or without peginterferon-alfa in patients with hepatitis $C$ virus genotype 3 infection and treatment-experienced patients with cirrhosis and hepatitis $C$ virus genotype 2 infection. Gastroenterology. 2015;149:1462-1470.

6. Afdhal N, Zeuzem S, Kwo P, et al. Ledipasvir and sofosbuvir for untreated HCV genotype 1 infection. N Engl J Med. 2014;370:1889-1898.

7. Zeuzem S, Dusheiko GM, Salupere R, et al. Sofosbuvir and ribavirin in HCV genotypes 2 and 3. N Engl J Med. 2014;370:1993-2001.

8. http://www.gilead.com/news/press-releases/2014/9/gilead-announces-generic-licensing-agreements-to-increase-access-to-hepatitis-c-treatments-in-developing-countries.

9. http://www.medicinespatentpool.org/the-medicines-patent-pool-signs-first-sub-licences-for-hepatitis-c-medicine-daclatasvir/

10. Manns MP, Czaja AJ, Gorham JD, et al. Diagnosis and management of autoimmune hepatitis. Hepatology. 2010;51:2193-2213.

11. Roberts EA, Schilsky ML. American Association for Study of Liver Diseases (AASLD). Diagnosis and treatment of Wilson disease: an update. Hepatology. 2008;47:2089-2111.

12. Malinchoc M, Kamath PS, Gordon FD, Peine CJ, Rank J, ter Borg PC. A model to predict poor survival in patients undergoing transjugular intrahepatic portosystemic shunts. Hepatology. 2000;31:864-871.

13. Towards elimination of viral hepatitis by 2030. Lancet. 2016;388:308.

14. Ghinea N, Lipworth W, Day R, Hill A, Dore GJ, Danta M. Importation of generic hepatitis $C$ therapies: bridging the gap between price and access in high-income countries. Lancet. 2017;389:1268-1272.

15. Vasudevan S, Shalimar Kavimandan A, Kalra N, Nayak B, Thakur B, et al. Demographic profile, host, disease \& viral predictive factors of response in patients with chronic hepatitis $C$ virus infection at a tertiary care hospital in north India. Indian J Med Res. 2016;143:331-340.

16. Sood A, Midha V, Mahajan R, et al. Results of sofosbuvir-based combination therapy for chronic hepatitis $\mathrm{C}$ cohort of Indian patients in real-life clinical practice. J Gastroenterol Hepatol. 2017;32:894-900.

17. Sidhu SS, Malhi NS, Goyal O, et al. Treatment of chronic hepatitis C genotype 3 with Sofosbuvir-based therapy: a real-life study. Hepatol Int. 2017:11:277-285.

18. Azam Z, Shoaib M, Javed M, Sarwar MA, Shaikh H, Khokhar N. Initial results of efficacy and safety of Sofosbuvir among Pakistani Population: a real life trial - Hepatitis Eradication Accuracy Trial of Sofosbuvir (HEATS). Pak J Med Sci. 2017;33:48-52.

19. Lawitz E, Mangia A, Wyles D, et al. Sofosbuvir for previously untreated chronic hepatitis C infection. N Engl J Med. 2013;368:1878-1887.

20. Flisiak R, Pogorzelska J, Flisiak-Jackiewicz M. Hepatitis C: efficacy and safety in real life. Liver International. 2017;37(Suppl 1):26-32.

21. Younossi ZM, Park H, Gordon SC, et al. Real-world outcomes of ledipasvir/sofosbuvir in treatment-naive patients with hepatitis C. Am J Manag Care. 2016;22(6 Spec No.):SP205-SP211.

22. Zeng Q-L, Xu G-H, Zhang J-Y, et al. Generic ledipasvir-sofosbuvir for patients with chronic hepatitis C: a real-life observational study. J Hepatol. 2017;66:1123-1129.

23. Alonso S, Riveiro-Barciela M, Fernandez I, et al. Effectiveness and safety of sofosbuvir-based regimens plus an NS5A inhibitor for 
patients with HCV genotype 3 infection and cirrhosis. Results of a multicenter real-life cohort. J Viral Hepat. 2017;24:304-311.

24. Chang CY, Nguyen P, Le A, et al. Real-world experience with interferonfree, direct acting antiviral therapies in Asian Americans with chronic hepatitis $C$ and advanced liver disease. Medicine. 2017;96:e6128.

25. Welzel TM, Petersen J, Herzer K, et al. Daclatasvir plus sofosbuvir, with or without ribavirin, achieved high sustained virological response rates in patients with HCV infection and advanced liver disease in a real-world cohort. Gut. 2016;65:1861-1870.

26. Elsharkawy A, Fouad R, El Akel W, et al. Sofosbuvir-based treatment regimens: real life results of 14409 chronic HCV genotype 4 patients in Egypt. Aliment Pharmacol Ther. 2017;45:681-687.

\section{SUPPORTING INFORMATION}

Additional Supporting Information may be found online in the supporting information tab for this article.

How to cite this article: Gupta S, Rout G, Patel AH, et al. Efficacy of generic oral directly acting agents in patients with hepatitis C virus infection. J Viral Hepat. 2018;25:771-778. https://doi.org/10.1111/jvh.12870 